\title{
Axially Symmetric Flow with Finite Cavities II
}

\author{
P. BROCK
}

An axially symmetric cavity flow of an ideal fluid is moving around an obstacle. The flow is either in a cylindrical pipe or an unbounded region and the cavity may be finite. Essentially is the assumption that the obstacle is star-like with respect to some point on the axis of symmetry. The existence of such flows was proved by the author in Part I. In the present Part II the behaviour of the free boundaries near the end-point on the axis of symmetry (in the case of a finite cavity) and near infinity (in the case of an infinite cavity) are investigated.

Key words: Non-continuous functional, symmetrization, arially symmetric flow AMS subject classification: $35 \mathrm{~J} 85$

\section{Introduction}

A three-dimensional axially symmetric cavity flow of an incompressible inviscid fluid is moving around an obstacle. In its shadow a cavity occurs which is bounded by a free surface and on which the modulus of the velocity is constant. We consider the two cases that the flow is either in a cylindrical pipe or in an unbounded region and make the geometrical assumption that the obstacle is star-like with respect to some point on the axis of symmetry. The existence of such flows was proved in Part I of this paper [2]. In the constructed solutions the free stream-lines are also star-like. Moreover it appears that the cavity is finite, i.e. the free stream-lines end on the axis of symmetry. In this paper we investigate the behaviour of the free stream-lines near infinity (in the case of an infinite cavity) or near their end-point (in the case of a finite cavity), respectively.

\section{Smooth fit of the free boundary on the axis of symmetry}

First we introduce some notations. If $X=(x, y)$ is a point in $\mathbf{R}^{2}$, then let $(r, \varphi)$ denote its polar coordinates. $B_{R}(X)$ denotes the interior of a ball with centre in $X$ and radius $R$, and we set $B_{R}=B_{R}(O)$, where $O$ is the origin $(0,0)$. We take $\{y=0\}$ as the axis of symmetry and introduce a finite and continuous curve $N$ (the obstacle), which is star-like with respect to the origin and has a representation $(a \geq 0, b>0)$

$$
\begin{aligned}
& N: X=X_{0}(t)=\left(x_{0}(t), y_{0}(t)\right) \text { for } 0 \leq t \leq a ; \\
& X_{0}(0)=(-b, 0), y_{0}(t)>0 \text { if } 0<t \leq a ;
\end{aligned}
$$

P. Brock: Universitat Leipzig, Institut für Mathematik, Augustuspl. 10, D - 04109 Leipzig ISSN $0232-2064 \% \$ 2.50$ C Heldermann Verlag Berlin 
$X_{0}$ is piecewise of class $C^{1+\alpha}, 0<\alpha<1, \quad \nabla X_{0}(t \pm 0) \neq 0$ for $0 \leq t \leq a$.

We choose $h>\max \left\{y_{0}(t): 0 \leq t \leq a\right\}$ and set $A=X_{0}(a)=\left(x_{A}, y_{A}\right), B=\{y=h\}$ and

$$
S=(\{x<-b\} \cap\{y=0\}) \cup N \cup \overline{A O} \cup(\{x>0\} \cap\{y=0\}) .
$$

By $\Omega$ we denote the domain between the curves $S$ and $H$ and by $\Omega_{\infty}$ the domain above $S$ (see Figures' 1,2).

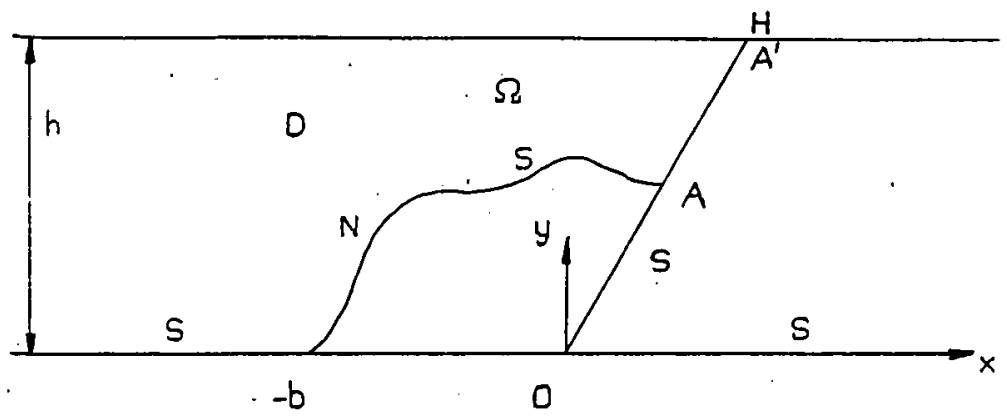

Figure 1

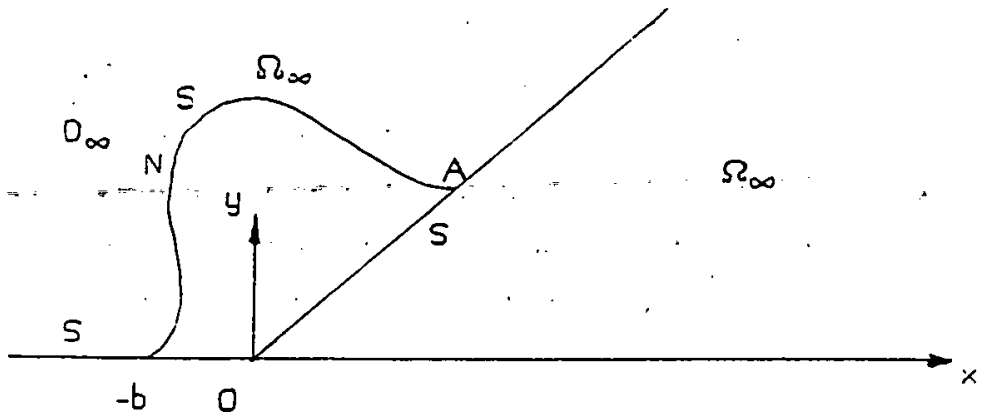

Figure 2

We summarize the main results of Part I [2] in the following two theorems.

Theorem 2.1: There are a function $u$, a number $\lambda>0$ and a curve $\Gamma \subset \Omega$, which has a representation

$$
\begin{aligned}
& \Gamma: r=R(\varphi) \text { for all } \varphi \in\left(0, \varphi_{A}\right), \text { with } \varphi_{A}=\arctan \frac{y_{A}}{x_{A}} \\
& R \in C^{\infty}\left(0, \varphi_{A}\right), \text { with } \lim _{\varphi \rightarrow 0} R(\varphi)=d \in(0,+\infty)
\end{aligned}
$$

such that

$$
0 \leq u(x, y) \leq \frac{y^{2}}{h^{2}}, L u:=u_{x x}+u_{y y}-\frac{u_{y}}{y} \geq 0 \text { in } \Omega \text { in the distribution sense, }
$$




$$
\begin{aligned}
& u=1 \text { on } B,\{u>0\}=: G \subset \Omega, \partial G \cap \Omega=\Gamma, u=0 \text { on } \Gamma \cup(\partial G \cap S), \\
& u \in C^{2}(G) \cap C(\bar{G}), \quad L u=0 \text { in } G, \\
& \frac{1}{y} \frac{\partial u}{\partial \nu}=\lambda \text { on } \Gamma \quad(\nu-\text { inner normul }) .
\end{aligned}
$$

In the case $d=+\infty$ (infinite cavity) $\Gamma$ has for great $x>0$ a representation $y=f(x)$ with $\lim _{x \rightarrow+\infty} f(x)=\sqrt{h^{2}-\frac{2}{\lambda}}$ and $\lambda \geq \frac{2}{h^{2}}$. In the case $d<+\infty$ (finite cavity) we have $\lambda<\frac{2}{h^{2}}$.

Theorem 2.2: There are a function $u$, a number $\lambda>0$ and a curve $\Gamma \subset \Omega_{\infty}$, which has a representation of the form (2), such that

$$
\begin{aligned}
& 0 \leq u \leq \frac{y^{2}}{2} \text { in } \Omega_{\infty}, \\
& G \subset \Omega_{\infty}, \quad \partial G \subset \Omega_{\infty}=\dot{\Gamma}, \quad u=0 \text { on } \Gamma \cup(\partial G \cap S),
\end{aligned}
$$

and the conditions (3) and (4) of the previous theorem are satisfied. Further we have $\lambda<1$ if the cavity is finite and $\lambda=1$ if the cavity is infinite .

The next lemma will be useful for us in the following .

Lemma 2.3: Let be $\varphi_{0} \in(0, \pi)$ and $\Sigma:=B_{r_{0}} \cap\left\{0<\varphi<\varphi_{0}\right\}, \tau_{0}>0$. Further let $v$ be a solution of the following boundary value problem $(C>0)$ :

$$
\begin{aligned}
& v=0 \text { on } \partial \Sigma \cap B_{r_{0}}, v(z(r, \varphi), y(r, \varphi))=: g(r, \varphi) \leq C \sin ^{2} \varphi \text { on } \partial \Sigma \cap \partial B_{r_{0}}, \\
& g \in C(\bar{\Sigma}), v \in C^{2}(\Sigma) \cap C\left(\bar{\Sigma} \backslash\left\{\left(\cos \varphi_{0}, \sin \varphi_{0}\right)\right\}\right), L v=0 \text { in } \Sigma .
\end{aligned}
$$

Then there are numbers $C_{1}, \alpha>0$ and a function $w \in C^{2}\left[0, \varphi_{0}\right]$ such that

$$
v(x(r, \varphi), y(r, \varphi)) \leq C_{1} r^{2+\alpha} w(\varphi) \sin ^{2} \varphi \text { in } \Sigma
$$

Proof: The idea is to choose $C_{1}, \alpha$ and $w$ such that

$$
g(r, \varphi) \leq C_{1} r^{2+\alpha} w(\varphi) \sin ^{2} \varphi \text { on } \partial \Sigma
$$

and $L_{1}\left(r^{2+\alpha} w(\varphi) \sin ^{2} \varphi\right) \leq 0$ in $\Sigma$, where $L_{1}:=\frac{\partial^{2}}{\partial r^{2}}+\frac{1}{r^{2}}\left(\frac{\partial^{2}}{\partial \varphi^{2}}-\cot \varphi \frac{\partial}{\partial \varphi}\right)$ denotes the represen. tation of the operator $L$ in polar coordinates. Then the inequality (6) follows by the maximum principle. Now letting $w(\varphi)=\exp \left(-\varepsilon \tan ^{2}\left(\frac{\varphi}{2}\right)\right)(\varepsilon, \alpha>0)$ we get

$$
\begin{aligned}
L_{1}\left(r^{2+\alpha} w(\varphi) \sin ^{2}(\varphi)=\right. & r^{\alpha} w(\varphi) \sin ^{2}(\varphi) \times \\
& \left(3 \alpha+\alpha^{2}-2 \varepsilon+\varepsilon^{2} \tan ^{6} \frac{\varphi}{2}+2 \varepsilon^{2} \tan ^{4} \frac{\varphi}{2}+\left(e^{2}-2 \varepsilon\right) \tan ^{2} \frac{\varphi}{2}\right) .
\end{aligned}
$$

The term on the right-hand side is non-positive in $\Sigma$ if we set $3 \alpha+\alpha^{2} \doteq \epsilon=\cos ^{6} \frac{\varphi_{0}}{2} \sin ^{-2} \frac{\varphi_{0}}{2}$

Lemma 2.4: Let be $(u, \lambda, \Gamma)$ be a triplet satisfying the conditions of Theorem 2.1 or 2.2 . 
Then there is a number $R=R(\lambda)>0$ such that $u=0$ in $B_{R} \cap \Omega$ or $B_{R} \cap \Omega_{\infty}$, respectively .

Proof First let $(u, \lambda, \Gamma)$ be a triplet satisfying the conditions of Theorem 2.1 and $X_{0}=$ $\left(x_{0}, y_{0}\right)$ a point in $\Omega \cap\left\{0<\varphi<\varphi_{A}\right\}$. We assume that $u\left(X_{0}\right)>0$. Then by [1: Lemma 5.2] we conclude for any $r$ with $r \leq \frac{y_{0}}{2}$ and $B_{r}\left(X_{0}\right) \subset \Omega$ that $\sup _{\partial B_{r}\left(X_{0}\right) \cap n} u \geq c y_{0} \lambda r$, where the constant $c$ is positive and independent of $\lambda$. There is a point $X_{1}=\left(x_{1}, y_{1}\right)$ on $\partial B_{\nu_{0} / 2}\left(X_{0}\right)\left(x_{1}=\right.$ $\left.r_{1} \cos \varphi_{1}, y_{1}=r_{1} \sin \varphi_{1}\right)$ such that $u\left(X_{1}\right) \geq \frac{c}{2} y_{0}^{2} \lambda$. Now we choose a number $\varepsilon_{0}>0$ such that $u=0$ on $B_{e_{0}} \cap \overline{O A^{\prime}}$. Because of (3) we can apply the previous lemma on the function $u$ in $B_{e_{0}} \cap \Omega$. This yields by the maximum principle

$$
u(x(r, \varphi), y(r, \varphi)) \leq C_{1} r^{2+\alpha} \sin ^{2} \varphi \text { in } B_{e 0} \cap \Omega,
$$

where the constant $C_{1}>0$ does not depend on $(r, \varphi)$. If we choose $C_{1}$ great enough, the same inequality (8) is valid in $\Omega \backslash B_{e_{0}}$. Therefore $u\left(X_{1}\right) \leq C_{1} r_{1}^{2+\alpha} \sin ^{2} \varphi_{1}=C_{1} y_{1}^{2} r_{1}^{\alpha}$. Because of $y_{1} \leq \frac{3}{2} y_{0}$ and $r_{1} \leq 3 r_{0}\left(x_{0}^{2}+y_{0}^{2}=r_{0}^{2}\right)$, we get now $c y_{0}^{2} \lambda \leq \frac{9}{2} C_{1} y_{0}^{2}\left(3 r_{0}\right)^{\alpha}$. Hence there is a number $C_{3}>0$, independent of $\lambda$ and $X_{0}$, such that $C_{3} \lambda \leq r_{0}^{\alpha}$. Choosing $R=\left(C_{3} \lambda\right)^{1 / \alpha}$ the assertion follows .

The proof is analogue if $(u, \lambda, \Gamma)$ is a triplet satisfying the conditions of Theorem 2.2. We only have to replace the domain $\Omega$ by $\Omega_{\infty}$ and the inequality $u(x, y) \leq y^{2}$ by (5)

Now we formulate the main result of the section .

Theorem 2.5: Let $(u, \lambda, \Gamma)$ be a triplet satisfying the conditions of Theorem 2.1 or 2.2 . Then $\partial\{u>0\}$ is of class $\bar{C}^{1}$ in a neighbourhood of the end-point of the free boundary.

Proof: The proof is in three steps. In the first step we show that

$$
\underset{\varphi \backslash 0}{\liminf _{\varphi}} \frac{R(\varphi)-R(0)}{\varphi}=-\infty \text {. }
$$

Assume that (9) is not true. Then there is a non-horizontal straight line $K$ through the endpoint $E=\left(x_{0}, 0\right)$ of the free boundary such that $u=0$ on $K \cap \Omega \cap B_{e}(E)$ for a small $\varepsilon>0$. Since the operator $L$ is homogeneous in $x$, we can prove as in the previous lemma the existence of a number $R<0$ such that $u=0$ in $B_{R}(E) \cap \Omega$. But this is impossible.

In the second step we prove

$$
\lim _{\varphi \downarrow 0} \frac{R(\varphi)-R(0)}{\varphi}=-\infty \text {. }
$$

To this, we consider functions $u_{\alpha}(X):=\frac{u(\alpha X+E)}{\alpha^{2}}, \alpha>0,(\alpha X+E) \in\{u>0\}$. Because of (9) there is a sequence $\left\{X_{n}\right\}_{n \geq 1} \subset \Gamma, X_{n}=\left(x_{n}, y_{n}\right), x_{n}<x_{0}$, with $X_{n} \rightarrow E$ and $\frac{y_{n}}{x_{n}-x_{0}} \longrightarrow 0$. We set $\alpha_{n}:=x_{0}-x_{n}$. Since $|\nabla u(X)| \leq C y, X \in B_{\varepsilon}(E) \cap \Omega$ for a constant $C>0$ and $\varepsilon>0$ small (see the proof of [1: Theorem 5.4]), it follows also $|\nabla u(X)| \leq C y, X \in B_{2} \cap\{y \geq 0\}$, for 
large $n$, with the same constant $C$. Therefore there are a subsequence $\left\{\alpha_{n}^{\prime}\right\}_{n \geq 1}$ and a function $u_{0} \in C^{0,1}\left(B_{2} \cap\{y \geq 0\}\right)$ such that $u_{\alpha_{n}^{\prime}} \rightarrow u_{0}$, uniformly in $B_{2} \cap\{y \geq 0\}$. We want to show that

$$
u_{0}(X)=\frac{\lambda}{2} y^{2} \quad \text { in } \quad B_{2} \cap\{y \geq 0\}
$$

We have $\partial\left\{u_{\alpha_{n}^{\prime}}>0\right\} \ni\left(-1, y_{n}^{\prime} / \alpha_{n}^{\prime}\right) \longrightarrow(-1,0)$ as $n \rightarrow+\infty$. Remember that $\Gamma$ is star-like and cannot oscillate near $\{y=0\}$ (see [1: Theorem 6.1]). Therefore there is a (small) number $\epsilon>0$ such that for any $p$ with $0<p<\varepsilon$ we get $u_{\alpha_{n}^{\prime}}>0$ in $B_{\frac{p}{2}}\left(-\frac{1}{2}, p\right)$ if $n$ is large enough. Moreover, in view of [1: Lemma 5.2] we conclude that $u_{\alpha_{n}^{\prime}} \geq c_{1} \lambda p^{2}$ in $B_{p / 4}\left(-\frac{1}{2}, p\right)$; where the number $c_{1}>0$ depends only on $p$. Therefore it follows $u_{0} \geq c_{1} \lambda p^{2}$ in $B_{p / 4}\left(-\frac{1}{2}, p\right)$. Since the number $p>0$ was arbitrary we get $u_{0}\left(-\frac{1}{2}, y\right)>0$ for any $y \in(0, \varepsilon)$. Further we have

$$
\frac{\partial}{\partial r} u(x(r, \varphi), y(r, \varphi)) \geq 0 . \text { in } \Omega
$$

therefore $\frac{\partial u_{0}}{\partial x} \geq 0$ in $B_{2} \cap\{y \geq 0\}$, and finally

$$
u_{0}>0: \text { in } \dot{B}_{2} \cap\left\{0<y<\frac{\varepsilon}{2}\right\} \cap\left\{x>-\frac{1}{2}\right\}
$$

It follows that $\{y=, 0\} \cap\left\{-\frac{1}{2}<x<0\right\} \subset\left\{u_{0}>0\right\}$ and

$$
\lim _{y \rightarrow 0} \frac{1}{y} \nabla u_{0}(x, y)=(0, \lambda) \text { for any } x \in\left(-\frac{1}{2}, 0\right) \text {, }
$$

since the free boundaries $\partial\left\{u_{\alpha_{n}^{\prime}}>0\right\} \cap\left\{-\frac{1}{2}<x<0\right\}$ converge to the segment $\{y=0\} \cap\left\{-\frac{1}{2}<\right.$ $x<0\}$. Because of (13) we have $L u_{0}=0$ in $B_{2} \cap\left\{0<y<\frac{e}{2}\right\} \cap\left\{x>-\frac{1}{2}\right\}$. Together with (14) this yields (11). Now for any $x>x_{0}$. we set $V(x)=\lim _{y \downarrow 0} \frac{1}{y} u_{y}(x, y)$. Because of (12) we have $V^{\prime}(x)^{\prime} \geq 0$ if $x>x_{0}$. Therefore the limit $\lim _{e_{1} \varepsilon_{0}} V(\dot{x})=\therefore V_{0}$ exists and we get $\lim _{y 10} \frac{1}{y} \frac{\partial}{\partial y} u_{0}(x, y)=V_{0}$ if $x>0$. In view of (14) it follows $V_{0}=\lambda$. Let $\left\{X_{n}\right\}_{n \geq 1}$ be a sequence of free boundary points with $X_{n} \rightarrow E$ and $\frac{v_{n}}{x_{0}-x_{n}} \geq \tau>0$. Fór this sequence we again consider the above defined functions $u_{\alpha_{i n}}$. There is a subsequence. $\left\{u_{\alpha_{a}^{\prime}}\right\}_{n \geq 1}$ such that $u_{\alpha_{i}^{\prime}} \longrightarrow u_{0}$.uniformly in $B_{2} \cap\{y \geq 0\}, \frac{x_{n}^{\prime}}{\alpha_{n}} \rightarrow C \geq \tau$ and $u_{0}(-1, C)=0$. On the other hand we can show as before that $\lim _{y \mid 0} \frac{\partial}{\partial_{y}} u_{0}(x, y)=\lambda$ for any $x^{\prime}>0$ and $u_{0}(X)=\frac{\lambda}{2} y^{2}$ by unique continustion, which is impossible. This proves (10).

In the third step we show that $\theta\{u>0\}$ is continuously differentiable in a neighbourhood of $E$. Again we consider a sequence of free boundary points $\left\{X_{n}\right\}_{n \geq 1}$ with $X_{n} \longrightarrow E$. We define a sequence of functions $\left\{u_{n}\right\}_{x \geq 1}$ by

$$
u_{n}(X)=\frac{1}{y_{n}^{2}} u\left(y_{n} X+\left(x_{n}, 0\right)\right), \quad\left(y_{n} X+\left(x_{n}, 0\right)\right) \in\{u>0\}
$$


There is a function $w_{0} \in C^{0,1}(\{y \geq 0\})$ satisfying the following properties :

$$
\begin{aligned}
& w_{0}(X) \geq 0 \text { in }\{y \geq 0\}, \quad L w_{0}=0 \text { in }\left\{w_{0}>0\right\} \cap\{y \geq 0\} \\
& \frac{1}{y} \frac{\partial w}{\partial \nu}=\lambda \text { on } \partial\left\{w_{0}>0\right\} \quad(\nu-\text { inner normal }), \quad(0,1) \in \partial\left\{w_{0}>0\right\}
\end{aligned}
$$

such that for a subsequence $\left\{u_{n}^{\prime}\right\}_{n \geq 1}$ we get $u_{n}^{\prime} \longrightarrow w_{0}$ uniformly in compact subsets of $\{y \geq 0\}$, and the free boundary $\partial\left\{w_{0}>0\right\}$ is locally analytic. We consider the speed function $q(X):=$ $\frac{1}{y}|\nabla u(X)|$. An easy calculation shows that $\nabla\left(y, \nabla\left(q^{2}\right)\right) \geq 0$ in $\{u>0\}$, i.e. $q$ is a subsolution. We can continue $q$ continuously on $\partial\{u>0\} \cap\{y=0\} \backslash\{E\}$. Setting $q=\lambda$ in $E$ we have $\lim _{X \in \Gamma, X \rightarrow E} q(X)=q(E)$. Since $q$ is bounded in a neighbourhood of $E$ we conclude as in [3: Lemms 6.7, p.184] that

$$
\lim _{x \in\{u>0\}, X \rightarrow E} q(X) \leq \lambda .
$$

It follows that $\frac{1}{y}\left|\nabla w_{0}(X)\right| \leq \lambda$ a.e. in $\left\{w_{0}>0\right\}$ and this implies that $\partial\left\{w_{0}>0\right\}$ is concave to $\left\{w_{0}>0\right\}$ (compare with [3: Lemma 11.2, p.327]). Further from $u_{r} \geq 0$ we get $\left(w_{0}\right)_{z} \geq 0$. It follows that $\partial\left\{w_{0}>0\right\}=\{y=1\}$ and $w_{0}(X)=\frac{\lambda}{2}\left(y^{2}-1\right)$ if $y \geq 1$. Thus the curves $\partial\left\{u_{n}^{\prime}\right\} \cap B_{\frac{1}{2}}(0,1)$ converge to $\{y=1\} \cap B_{\frac{1}{2}}(0,1)$ in the Hausdorff metric, i.e. for any $\varepsilon>0$ there is a number $n_{0}=n_{0}(\varepsilon)$ such that $\partial\left\{u_{n}^{\prime}\right\} \cap B_{\frac{1}{2}}((0,1)) \subset\{1-\varepsilon<y<1+\varepsilon\}$ for any $n \geq n_{0}$. But this is the flatness condition of [1: Theorem 8.1]. Therefore the tangents on $\partial\left\{u_{n}^{\prime}>0\right\}$ in $(0,1)$ converge to $\{y=1\}$. This finishes the proof of the theorem 1

Corollary 2.6: $\frac{1}{y} \nabla u(X)$ is uniformly continuous in a small neighbourhood of $E$.

Proof : It follows from the proof of the previous theorem that the function $\frac{u_{y}}{y}$ is continuous on $\theta\{u>0\} \cap B_{\varepsilon}(E), \varepsilon>0$ small. Then we can show as in [3: Theorem 11.3] that $\frac{u_{x}}{y}$ is uniformly continuous in $\{u>0\} \cap B_{e}(E)$. The same is true for the function $\frac{u_{x}}{y}$ in view of (16)

\section{Convergence of the free boundaries}

In this section we investigate the behaviour of the free boundaries near infinity.

Theorem 3.1: Let $(u, \lambda, \Gamma)$ be a triplet satisfying the conditions of Theorem 2.1 with an infinite cavity. Then

$$
\lim _{x \rightarrow+\infty} f^{\prime}(x)=0
$$

where $y=f(x)$ is the representation of $\Gamma$ from Theorem 2.1 .

Proof: Because of the above mentioned theorem there is a sequence of positive numbers $\left\{d_{n}\right\}_{n \geq 1}$ with $d_{n} \longrightarrow 0$ such that 
and the free boundary $\partial\left\{u_{\infty}>0\right\} \cap\{y>0\}$ is an $x$-graph. The function $v=e_{0} \nabla u_{\infty}-\lambda_{0} y$, where $e_{0}$ is the unit vector $\frac{1}{\lambda_{0}} \nabla u_{\infty}(0,1)$, satisfies

$$
\begin{aligned}
L v & =\Delta v-\frac{v_{y}}{y}=\frac{1}{y}\left(\lambda_{0}-\frac{1}{y}\left(u_{\infty}\right)_{y} e_{0}(0,1)\right) \\
& \geq \frac{1}{y}\left(\lambda_{0}-\frac{1}{y}\left|\nabla u_{\infty}\right|\right) \geq 0 \text { in } B_{2} \cap\{y>0\} .
\end{aligned}
$$

Since $v$ takes its maximum (zero) at the point $(0,1)$, the strong maximum principle gives $v \equiv 0$, that is $\nabla u_{\infty} e_{0}=\lambda_{0} y$. Recalling $\left|\nabla u_{\infty}\right| \leq \lambda_{0} y$ we get $\nabla u_{\infty}=\lambda_{0} y e_{0}$ and therefore, as $u_{\infty}(x, 0)=$ $0, u_{\infty}(X)=\frac{\lambda_{0}}{2}\left(y^{2}-c^{2}\right)$, where $c$ is an unknown constant with $0 \leq c \leq 1$. Comparing with (19) we see that $1 \geq \lambda_{0}$. Thus the function $q_{0}$ takes its maximum on the free boundary. Therefore $\Gamma_{0}$ is concave to the domain $\left\{u_{0}>0\right\}$. $\Gamma_{0}$ cannot have an end-point on the axis $\{y=0\}$, otherwise we could easily derive a contradiction to Theorem 2.5. It follows that $\Gamma_{0}=\{y=1\}$. Finally the property (18) was derived in [3: Theorem 21.1,p.405]

\section{REFERENCES}

[1] ALT, H.W. and L.A. CAFFARELLI: Existence and regularity for a minimum problem with free boundary. J. Reine Angew. Math. 325 (1981), 105 - 144.

[2] BROCK, F.: Axially symmetric flow with finite cavities I. Z. Anal. Anw, 12 (1993), $97-112$.

[3] FRIEDMAN, A.: Variational Principles and Freo Boundary Problems. New York: Wiley \& Sons 1982 . 